\title{
In vitro resorbability and granular characteristics of 3D-printed hydroxyapatite granules versus allograft, xenograft, and alloplast
}

\section{Kongkrit Taecha-apaikun}

Khonkaen University Faculty of Dentistry https://orcid.org/0000-0002-8640-2857

Poonsak Pisek ( $\nabla$ poonsak@kku.ac.th )

Faculty of Dentistry, Khon Kaen University https://orcid.org/0000-0001-5413-9321

Jintamai Suwanprateeb

MTEC: Metal and Materials Technology Center

Faungchat Thammarakcharoen

MTEC: Metal and Materials Technology Center

Uthaiwan Arayatrakoollikit

Khonkaen University Faculty of Dentistry

Thidarat Angwarawong

Khonkaen University Faculty of Dentistry

\section{Supaporn Kongsomboon}

Khonkaen University Faculty of Dentistry

\section{Araya Pisek}

Khonkaen University Faculty of Dentistry

\section{Research}

Keywords: Bone graft, 3D printing hydroxyapatite, Degradability, Wicking, Granular agglomeration

Posted Date: January 25th, 2021

DOl: https://doi.org/10.21203/rs.3.rs-153153/v1

License: (1) (1) This work is licensed under a Creative Commons Attribution 4.0 International License. Read Full License 
Title:

In vitro resorbability and granular characteristics of 3D-printed hydroxyapatite granules versus allograft, xenograft, and alloplast

Study design: Experimental study

Running Head (abbreviated title)

Comparative properties of 3DP HA granules

Authors:

1. Kongkrit Taecha-apaikun ${ }^{1}$,

2. Poonsak Pisek ${ }^{1}$,

3. Jintamai Suwanprateeb ${ }^{2}$,

4. Faungchat Thammarakcharoen ${ }^{2}$,

5. Uthaiwan Arayatrakoollikit ${ }^{3}$,

6. Thidarat Angwarawong ${ }^{4}$,

7. Supaporn Kongsomboon ${ }^{5}$,

8. Araya Pisek ${ }^{1}$ 
Authors' Affiliation:

${ }^{1}$ Department of Preventive Dentistry, Faculty of Dentistry, Khon Kaen University, Khon Kaen 40002, Thailand.

${ }^{2}$ Biofunctional Materials and Devices Research Group, National Metal and Materials Technology Center, National Science and Technology Development Agency, Pathumthani 12120, Thailand

${ }^{3}$ Department of Restorative Dentistry, Faculty of Dentistry, Khon Kaen University, Khon Kaen 40002, Thailand.

${ }^{4}$ Department of Prosthodontics, Faculty of Dentistry, Khon Kaen University, Khon Kaen 40002, Thailand.

${ }^{5}$ Department of Oral and maxillofacial Dentistry, Faculty of Dentistry, Khon Kaen University, Khon Kaen 40002, Thailand.

Corresponding author : Poonsak Pisek

FAX: +6643202862 E-mail: poonsak@kku.ac.th 


\begin{abstract}
Background: Three-dimensionally printed hydroxyapatite (3DP HA) was investigated in regards to its functional properties supporting bone regeneration and tooth movement. The material's high porosity and nanocrystal structure were investigated in terms of degradability, wicking property, and granular agglomeration in order to identify its potential for use as bone graft in alveolar cleft applications.
\end{abstract}

Materials and methods: Commercially available bovine xenograft (Bio-Oss), biphasic calcium phosphate alloplast (BoneCeramic), and two types of freeze-dried bone allograft granules (SureOss Plus and RegenOss) were employed as control samples for comparison. In vitro degradability was studied by submerging the samples in $\mathrm{pH} 7.4$ buffered solution at $37^{\circ} \mathrm{C}$ for 28 days and determining subsequent weight loss percentage. The wicking property and granular agglomeration were evaluated by putting the granules in contact with deionized water, whole blood, and phosphate-buffered saline (PBS).

Results: SureOss Plus and RegenOss showed the greatest weight loss at 28 days (6.64 and $8.91 \%$, respectively) followed by 3DP HA $(2.82 \%)$, Bio-Oss $(0.21 \%)$, and BoneCeramic (0.20\%). In contrast, 3DP HA showed significantly greater wicking ability than other samples for all types of tested liquids. SureOss Plus exhibited the greatest granular agglomeration for all liquid types followed by 3DP HA and Bio-Oss, Regenoss, and BoneCeramic, respectively.

Conclusions: 3DP HA was found to be a favorable candidate for bone grafting in alveolar cleft treatment. Its resorption ability and exceptionally high wicking ability would be beneficial in bone healing and tooth movement. In addition, its moderate granular agglomeration capacity would help in graft handling and manipulation. 
Keywords: Bone graft, 3D printing hydroxyapatite, Degradability, Wicking, Granular agglomeration 


\section{Background}

Alveolar cleft is an orofacial anomaly common in cleft lip and cleft palate patients and can cause several complications, such as impeded tooth eruption and position and swallowing and speech impairments. Treatment options vary and depend on a number of factors, but the main objectives tend to be to provide bone continuity to the maxillary arch and support for tooth eruption on the cleft side, eradicate the fitulas, and restore aesthetic appearance. Treatment of alveolar cleft has evolved over many years, with gingivoperiosteoplasty being common early on and bone grafting becoming the most widely accepted treatment today [1].

Generally, the use of autogenous bone grafts is considered to be the gold standard for alveolar bone grafting, as this type of graft possesses osteogenic capacity from existing bone cells, osteoinductive capacity from signaling molecules such as bone morphogenic proteins, and osteoconductive capacity from the hard tissue structure. These characteristics can help in triggering new bone formation [2]. However, the major disadvantages of harvesting autogenous bone, either intraorally or extraorally, are donor site morbidity, neurosensory disturbances, pain, and an increase in operating time [3]. Allografts or xenografts are alternatives, but they pose a risk of disease transmission, and their osteoinductivity and osteoconductivity can be compromised due to the treatment process used to ensure their safe application [4-5]. Alloplasts are synthetic materials that have been employed as alternatives to overcome all of the aforementioned problems. The most valuable aspect of alloplasts is their limitless supply for surgical procedures without the risk of donor site morbidity, disease transmission, or immunogenic reaction. A variety of materials have been developed as alloplasts, including polymers, ceramics, metals, and composites [6-7]. 
Calcium phosphate is one type of bioceramic material that has been used widely as a bone substitute due to comprising the main inorganic component of bone. The material is also advantageous because it can be categorized according to its calcium and phosphorus $(\mathrm{Ca} / \mathrm{P})$ ratio and crystal structure; for example, hydroxyapatite (HA), beta-tricalcium phosphate $(\beta-\mathrm{TCP})$, and biphasic hydroxyapatite/tricalcium phosphate $(\mathrm{BCP})$ are all types of calcium phosphate [8]. Conventionally, calcium phosphate has been shaped using traditional ceramic techniques, such as slip cast or die pressing, and other techniques have also been used, such as foam infiltration or freeze casting [9]. After precursors are formed into the desired structure, they are usually sintered at high temperatures to gain mechanical strength and densification. As a consequence, micropores (pores between each crystal) are reduced, as are bioactivity and serum absorption [10].

At present, 3D printing technology has been developed and is utilized for calcium phosphate fabrication. This can increase structural precision and the potential for customization. Various printing methods and materials have been proposed, but most require high-temperature sintering [11-12]. However, a 3D printed HA (3DP HA) that does not require sintering and which is processed at much lower temperatures was recently developed. The material contains high porosity and a microporous structure, known to be crucial factors in stimulating biological activity [13]. 3DP HA also possesses low crystallinity and nanosized crystals that closely mimic the natural hydroxyapatite crystal structure of bone tissue, identified as having a nano-sized plate-like structure [14]. Providing more surface area, nanosized HA crystals are considered to be more bioactive than larger-sized sintered HA crystals [15]. Due to these characteristics, 3DP HA has shown resorption ability in both in vitro and in vivo environments in contrast to non-resorbable, high-temperature sintered HA. The material has already had a positive outcome in a pilot clinical study investigating socket preservation [16-17]. 
A key property determining the suitability of a material for alveolar bone grafting is its porosity, which facilitates ingrowth of blood vessels and tissues [18]. Furthermore, an ideal bone graft for alveolar cleft should have high degradability or even display complete degradation in order to aid bone formation, provide rapid stabilization, and support orthodontic tooth movement into the cleft site [19-20]. The wicking ability of the bone graft, allowing it to absorb liquid such as blood at a rapid rate, can also be advantageous, as blood contains beneficial factors and components for osteogenesis [21]. In addition, high liquid absorption of graft granules, the most common form of bone graft, is useful in that the cohesive and adhesive forces between the liquid and material during absorption can act to bridge the bone graft granules by forming an agglomeration [22]. This enables surgeons to manipulate grafts easily by mixing graft granules with standard saline or blood [23].

It was envisaged that 3DP HA could potentially be applied as a bone graft material for alveolar cleft in cleft lip and cleft palate patients. Prior to clinical study, it is important to determine that 3DP HA has functional properties comparable to bone grafts typically used in this type of application. Hence, the present study was aimed at analyzing and comparing functional properties of 3DP HA versus several currently available bone grafts, including two types of freeze-dried bone allografts, one bovine xenograft, and one synthetic alloplast. Degradability and wicking ability, required for a viable alveolar bone grafting application, were examined, and granular agglomeration when in contact with liquids such as water, saline, or blood was also investigated to determine the ease of manipulation in a clinical setting. The knowledge gained from this study can be useful for justifying the use or non-use of 3DP HA, revealing its advantages and drawbacks, and also elucidating the precautions that should be taken when this material is used in clinical applications for bone grafting in alveolar cleft treatment. 


\section{RESULTS}

Figure 1 shows the granule morphology of each sample. 3DP HA had a connected and agglomerated granular structure consisting of macro- as well as numerous micro-pores. BioOss, SureOss Plus, and RegenOss were all prepared from natural bone. Their morphology thus resembled the structure of the bone used, either cortical or cancellous bone, after being crushed into granules. Bio-Oss had a trabecular structure comprising large interconnected pores with thick pore walls whereas SureOss Plus and RegenOss both displayed dense and elongated granules without macro-pores. However, an additional layer of CMC was observed on the surface of SureOss Plus. BoneCeramic granules had a broken trabecular-like structure comprising thin pore walls and sharp edges. Figure 2 shows the weight loss percentage of all samples after immersion in $\mathrm{pH} 7.4$ TRIS-buffered solution at 1, 7, 14, and 28 days. Weights of the Bio-Oss and BoneCeramic samples remained relatively unchanged throughout the study period. In contrast, the weights of 3DP HA, SureOss Plus, and RegenOss continuously decreased as immersion time increased. Initially, weight decreased rapidly (at the first $24 \mathrm{~h}$ ) and then subsequently decreased at a lower rate. Both SureOss Plus and RegenOss displayed relatively similar rates of weight loss over time and both had a greater degree of weight loss than 3DP HA. A multiple comparison test of weight loss percentages among the samples at day 28 revealed significant differences among the samples $(\mathrm{p}<0.05)$, as shown in Table 1. RegenOss displayed the greatest weight loss, followed by SureOss Plus and 3DP HA, respectively. All three weight losses were significantly greater than those of Bio-Oss and BoneCeramic, which showed limited change. No significant difference between Bio-Oss and BoneCeramic weight loss was noted. 


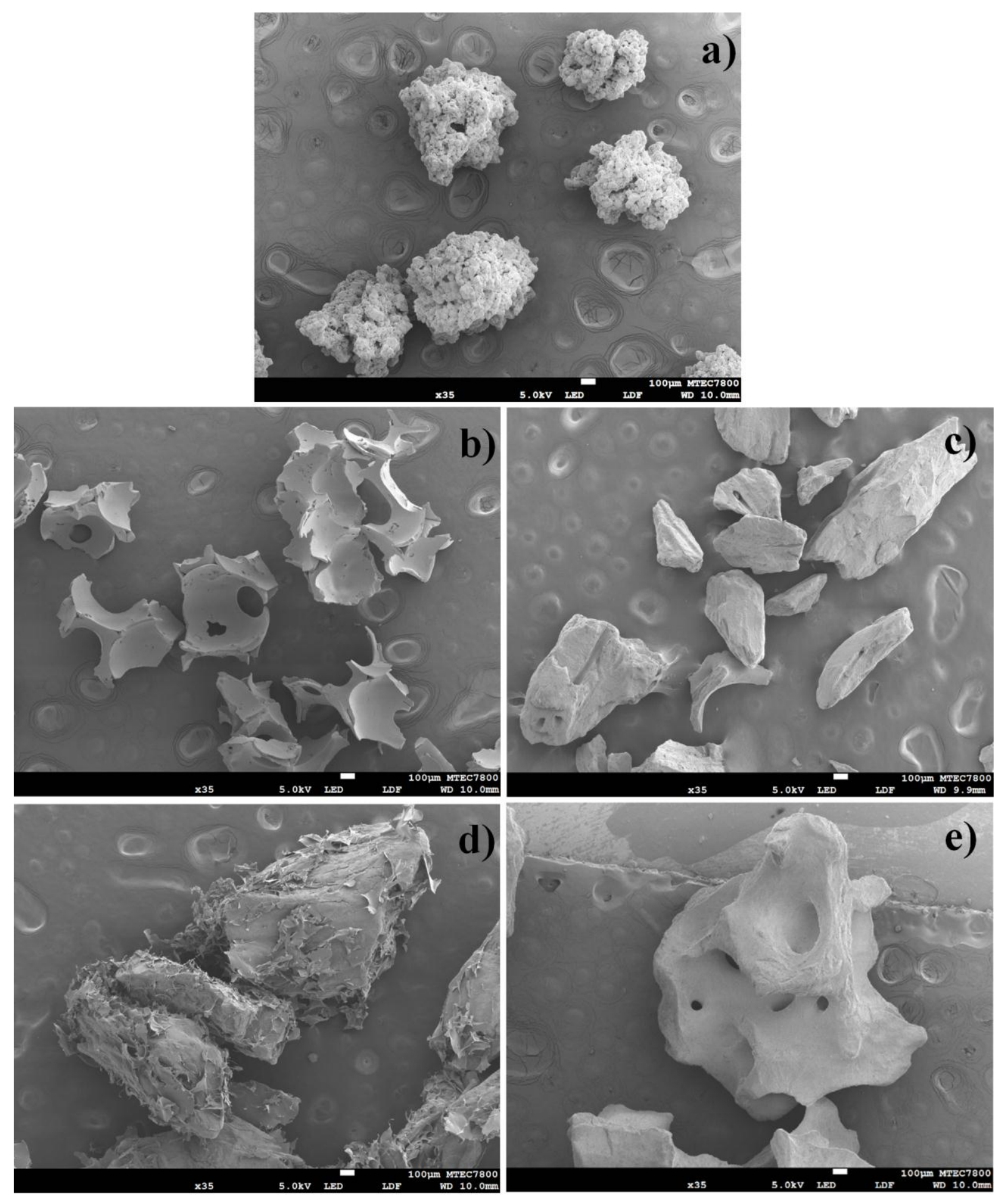

Figure 1. Morphology of the samples: a) 3DP HA; b) BoneCeramic; c) RegenOss; d)

SureOss Plus; and e) Bio-Oss. 


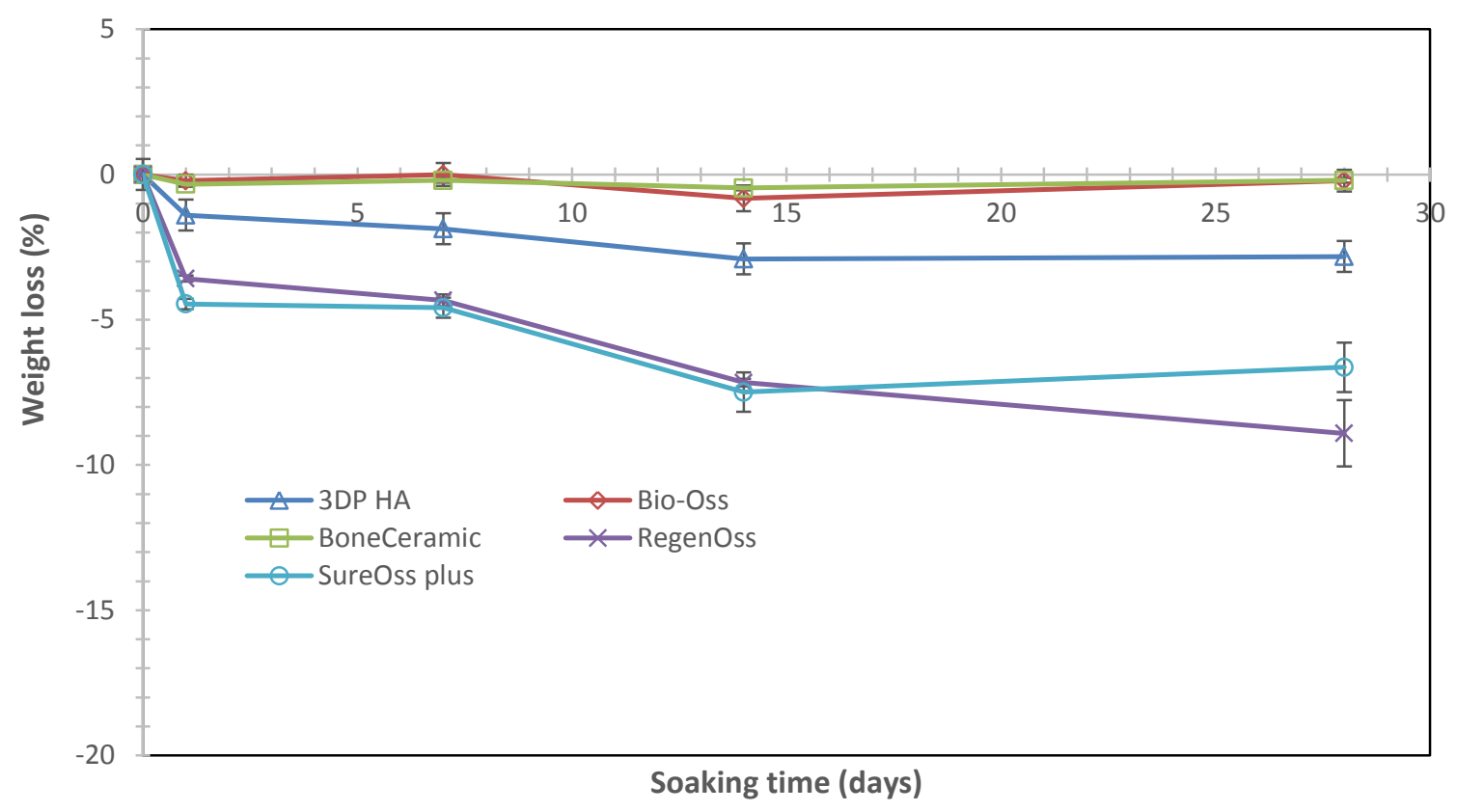

Figure 2. The change in weight loss percentage of samples at different periods after immersion in $\mathrm{pH} 7.4$ TRIS-buffered solution $(\mathrm{n}=3)$. Data are presented as the mean \pm standard deviation (SD).

Table 1. Findings from the multiple comparison test of the weight loss percentages at day 28 . Tukey's post hoc test was used, and data are presented as the mean \pm standard deviation (SD).

\section{Group 1 Group 2 Group 3 Group 4 Group 5 ANOVA Significant 3DP HA Bio-Oss RegenOss SureOss BoneCe ${ }^{\text {(p-value) }}$ difference \\ Plus ramic between groups}

$\begin{array}{cccccccc}\text { Weight loss } & 2.82 \pm 0.22 & 0.21 \pm 0.37 & 8.91 \pm 1.14 & 6.64 \pm 0.85 & 0.20 \pm 0.13 & <0.001 * & 1-2,1-3,1-4 \\ \text { percentage } & & & & & 1-5,2-3,2-4, \\ (\%) & & & & 3-4,3-5,4-5\end{array}$

*Statistically significant difference $(\mathrm{p}<0.05)$. 
Figure 3 shows a comparison of liquid absorption percentages over time among the samples when using deionized water (DI), whole blood, and phosphate-buffered saline (PBS). Regardless of the type of liquid used, the general liquid absorption characteristics of all samples were similar in that the absorption occurred quickly initially and subsequently continued at a lower rate. In the cases of DI and PBS (Figures 3.A-3.B), no difference was observed in either the liquid absorption ability or characteristics of the samples when paired with the different liquids. Among all of the samples, 3DP HA showed the greatest liquid absorption ability and the absorption persisted even at the end of the 180-second study period. Meanwhile, although Bio-Oss initially absorbed liquid quickly, similar to 3DP HA, absorption was subsequently limited and remained relatively unchanged. In the cases of SureOss Plus, RegenOss, and BoneCeramic, liquid absorption was substantially lower than that of 3DP HA and Bio-Oss, but that of SureOss Plus and RegenOss were slightly higher than BoneCeramic. When whole blood was used (Figure 3.C), different absorption characteristics were observed between BoneCeramic and Bio-Oss. BoneCeramic, which absorbed the least DI and PBS, absorbed more whole blood than Bio-Oss, which in fact achieved the least blood absorption. A multiple comparison test of the liquid absorption percentages at 180 seconds revealed significant differences among the samples $(p<0.05)$, as shown in Table 2. For all liquids tested, 3DP HA displayed the greatest liquid absorption, and the values were significantly greater than those of other samples. In the cases of DI and PBS, Bio-Oss displayed moderate liquid absorption. While its DI and PBS absorption ability was greater than that of RegenOss, SureOss Plus, and BoneCeramic, it was still significantly lower than that of 3DP HA. BoneCeramic demonstrated a greater blood absorption ability than RegenOss, SureOss Plus, and Bio-Oss, but no significant difference was observed. 
A. Deionized water

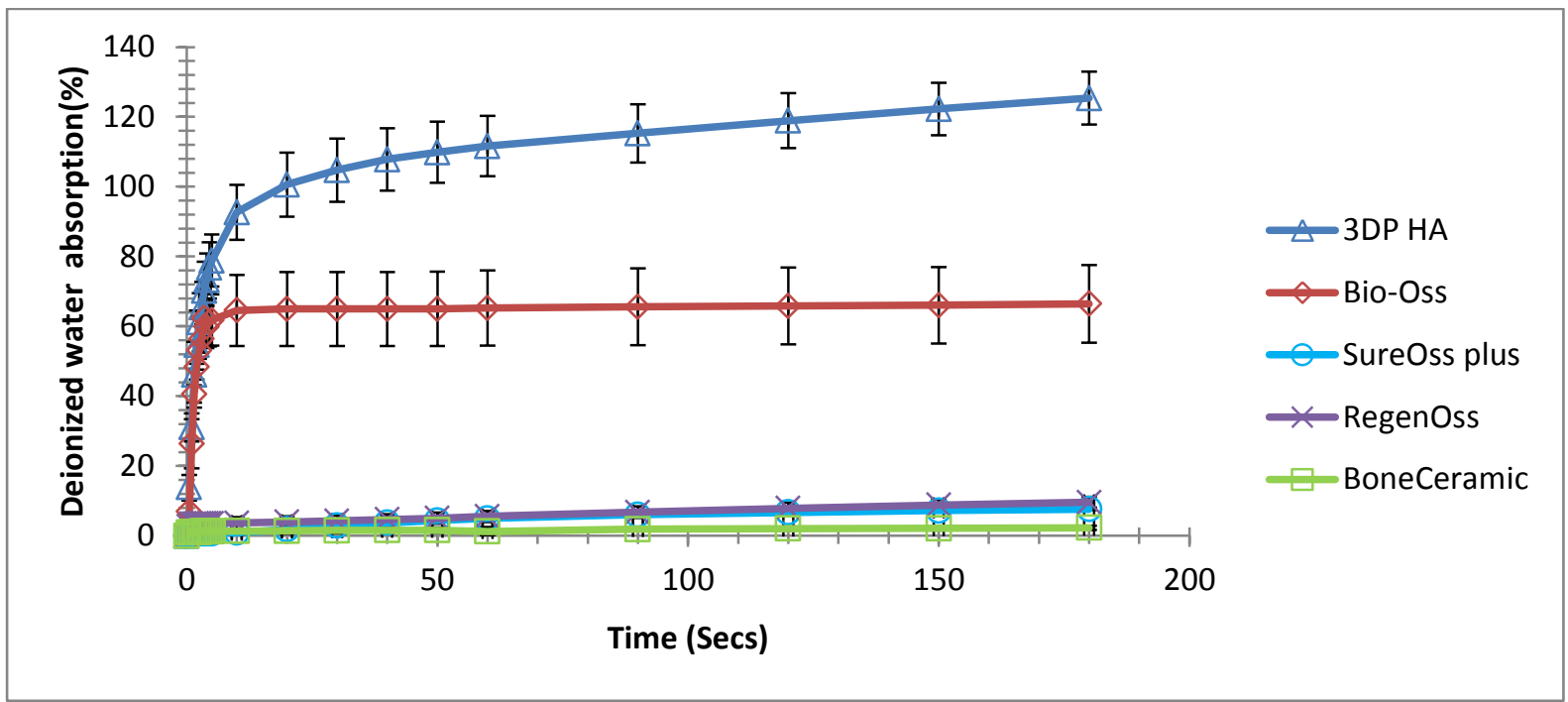

Figure 3. A, Changes in deionized water absorption percentage by samples over time $(n=3)$.

Data are presented as the mean \pm standard deviation (SD).

B. PBS

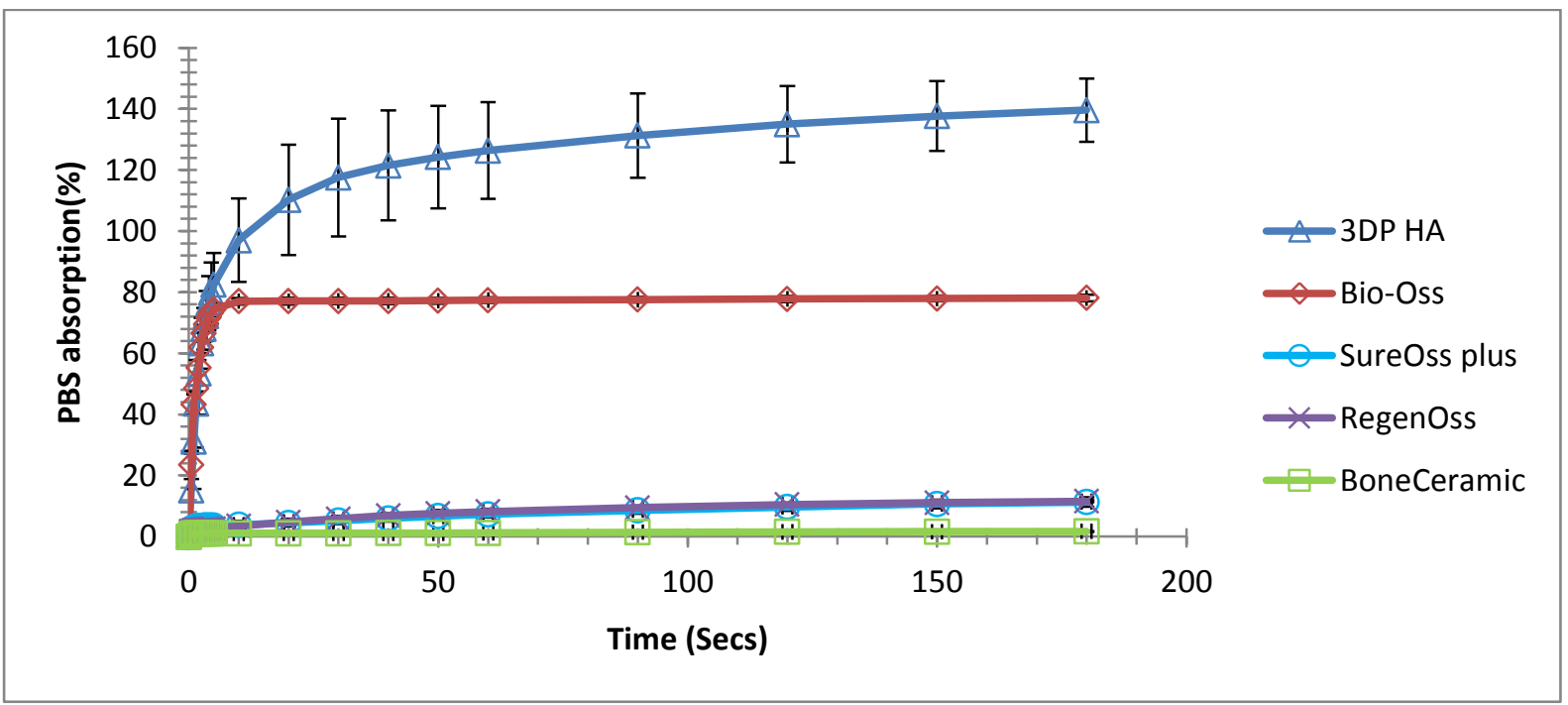

Figure 3. B, Changes in phosphate-buffered saline absorption percentage by samples over time $(n=3)$. Data are presented as the mean \pm standard deviation (SD). 
C. Whole blood

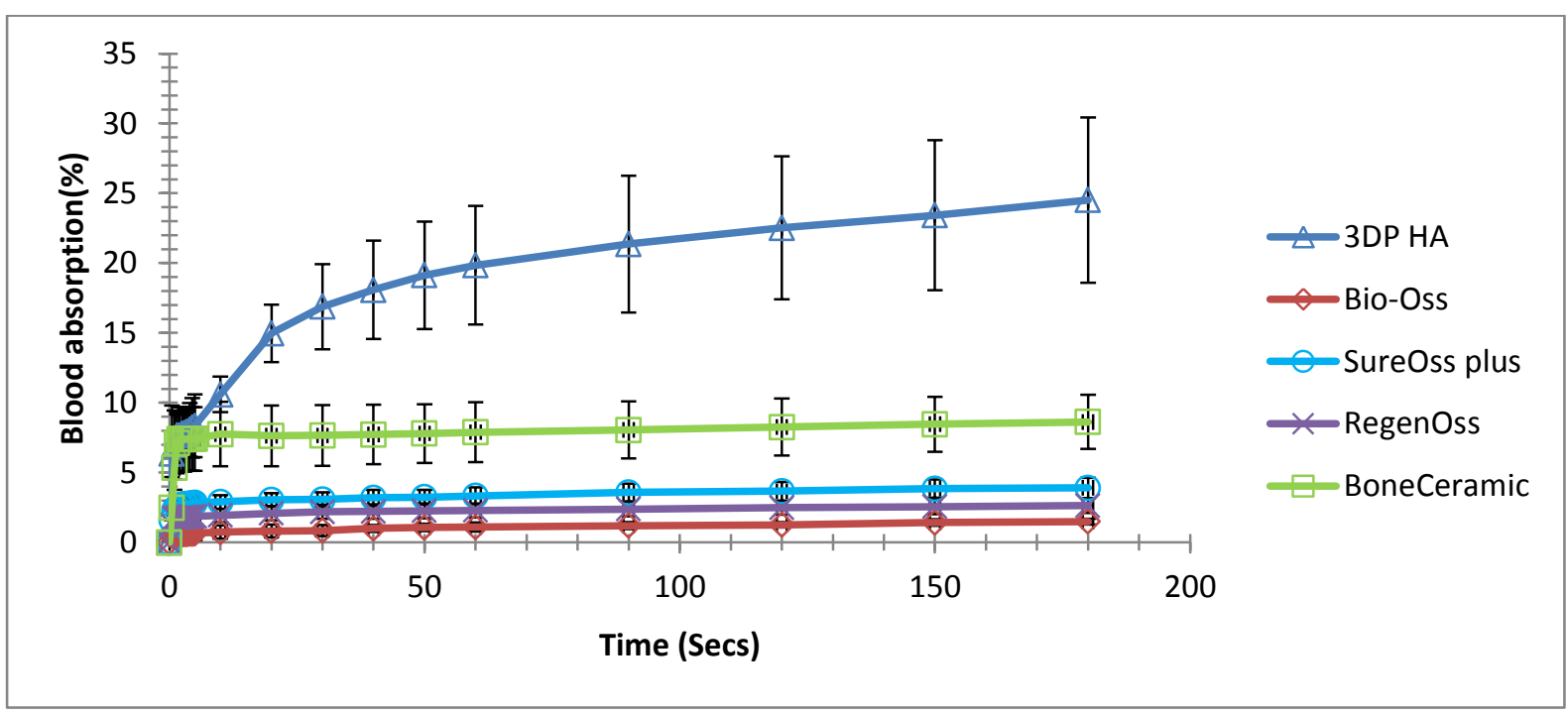

Figure 3. C, Changes in blood absorption percentage by samples over time $(n=3)$. Data are presented as the mean \pm standard deviation (SD).

Table 2. Findings from the multiple comparison test of the liquid absorption percentages at 180 seconds. Tukey's post hoc test was used, and data are presented as the mean \pm standard deviation (SD).

\begin{tabular}{ccccccc}
\hline Group 1 & Group 2 & Group 3 & Group 4 & Group 5 & ANOVA & Significant \\
3DP HA & Bio-Oss & RegenOss & SureOss & BoneCera & (p-value) & difference between \\
& & & Plus & mic & groups \\
& & & & & \\
\hline
\end{tabular}

\section{Liquid}

absorption

$(\%)$

DI $\quad 125.38 \pm 7.52 \quad 66.47 \pm 11.11 \quad 9.60 \pm 1.53 \quad 7.66 \pm 0.31 \quad 2.30 \pm 0.60 \quad<0.001 * \quad 1-2,1-3,1-4,1-5$, 


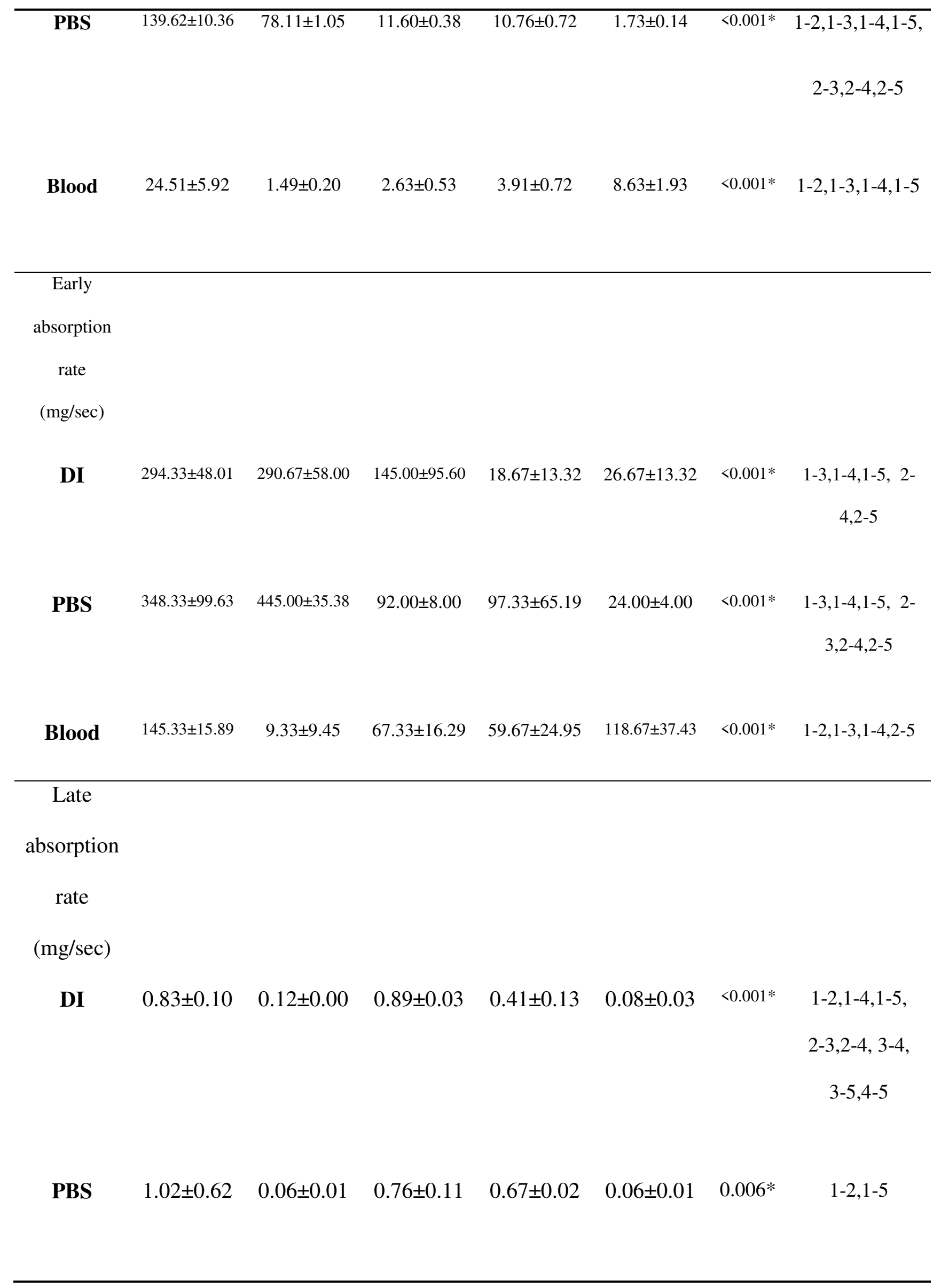




\begin{tabular}{llllllll}
\hline Blood & $0.36 \pm 0.13$ & $0.06 \pm 0.05$ & $0.08 \pm 0.01$ & $0.10 \pm 0.03$ & $0.09 \pm 0.03$ & $0.001 *$ & $1-2,1-3,1-4$
\end{tabular}

*Statistically significant difference $(\mathrm{p}<0.05)$.

Table 3 compares the agglomeration volume of the different samples when using deionized water (DI), whole blood, or phosphate-buffered saline (PBS). Regardless of the type of liquid used, SureOss Plus showed the greatest agglomeration volume after coming into contact with the liquids. 3DP HA and Bio-Oss also showed comparable agglomeration volumes to SureOss Plus when tested with DI, but the volume was significantly lower with PBS and whole blood. BoneCeramic and RegenOss showed the lowest and second-lowest agglomeration volume, respectively, when measured with DI and PBS. However, BoneCeramic was able to achieve a greater volume than RegenOss when tested with whole blood, for which the latter had the lowest agglomeration volume. The blood agglomeration volume of BoneCeramic was even comparable to 3DP HA and Bio-Oss.

Table 3. Findings from the multiple comparison test of the agglomeration volume. Tukey's post hoc test was used, and data are presented as the mean \pm standard deviation (SD).

\begin{tabular}{|c|c|c|c|c|c|c|c|}
\hline $\begin{array}{c}\text { Agglomera } \\
\text { tion }\end{array}$ & $\begin{array}{l}\text { Group } 1 \\
\text { 3DP HA }\end{array}$ & $\begin{array}{l}\text { Group } 2 \\
\text { Bio-Oss }\end{array}$ & $\begin{array}{l}\text { Group } 3 \\
\text { RegenOss }\end{array}$ & $\begin{array}{l}\text { Group } 4 \\
\text { SureOss }\end{array}$ & $\begin{array}{l}\text { Group } 5 \\
\text { BoneCera }\end{array}$ & $\begin{array}{l}\text { ANOVA } \\
\text { (p-value) }\end{array}$ & $\begin{array}{l}\text { Significant } \\
\text { difference }\end{array}$ \\
\hline $\begin{array}{l}\text { volume } \\
\left(\mathrm{mm}^{3}\right)\end{array}$ & & & & Plus & mic & & between groups \\
\hline DI & $144.76 \pm 15.74$ & $135.90 \pm 16.82$ & $78.67 \pm 6.43$ & $151.21 \pm 5.85$ & $30.91 \pm 3.64$ & $<0.001 *$ & $1-3,1-5,2-3,2-5$ \\
\hline
\end{tabular}


PBS

Whole $\quad 57.14 \pm 2.86 \quad 54.70 \pm 8.24 \quad 23.00 \pm 3.61 \quad 101.45 \pm 6.32 \quad 53.33 \pm 8.20 \quad<0.001 * \quad 1-3,1-4,2-3,2-4$, blood

*Statistically significant difference $(\mathrm{p}<0.05)$.

\section{DISCUSSION}

Several bone graft materials have been studied for use in alveolar bone grafting. Previous research has shown no difference in terms of new bone formation and clinical outcomes between bovine xenograft and autogenous bone when used for secondary alveolar bone grafting in patients with alveolar cleft [24]. When use of a mixture of hydroxyapatite and autogenous bone was compared with pure autogenous bone use for secondary alveolar bone grafting, patients did not show any complications. New bone formation and spontaneous canine eruption into the graft site were found to be similar between the two test groups [2526]. However, in terms of orthodontic tooth movement into the graft site, degradation ability is needed for the bone graft to resorb and to be replaced by new bone, and most hydroxyapatite has been shown to have a limited resorption ability and a slow degradation rate due to having undergone a high-temperature sintering process [27]. Therefore, hydroxyapatite with a greater resorption rate would be favorable and is required in such applications. 
In vitro resorbability of the samples in this study was determined by measuring their weight loss percentage subsequent to immersion in a liquid, a technique typically employed for in vitro assessment. Simulated body fluid (SBF) is generally used as a media in this test [16], but in this study the degree of resorption could be confounded by the re-precipitation process of the SBF's incorporated $\mathrm{Ca}$ and $\mathrm{P}$ ions, which could occur on the surface of the immersed samples. Specifically, the decrease in weight due to the resorption of the samples could be countered by the neo-formation of calcium phosphate crystals on their surface. This could result in an unchanged or even a re-increase of sample weights after certain times if the precipitation rate is equal to or greater than the resorption rate of the samples [16]. Therefore, $\mathrm{pH}$ 7.4 TRIS-buffered solution containing no $\mathrm{Ca}$ and $\mathrm{P}$ ions was employed as an alternative in order to evaluate the native resorbability of the samples without extrinsic influence. The results showed that both allografts (SureOss Plus and RegenOss) displayed the greatest weight loss percentages among the samples. This was not surprising since allograft is derived from natural bone, known to possess high resorption ability [28]. It should be noted that SureOss Plus showed a slightly greater weight loss percentage than RegenOss on the first day. Although they are both FDBA, SureOss Plus contains 5\% CMC in its composition. $\mathrm{CMC}$ is a water-soluble polymer; thus, the faster resorption rate of CMC relative to FDBA is likely responsible for the difference observed. 3DP HA was the second-most resorbed sample after the allografts. Bio-Oss and BCP, on the other hand, showed limited weight changes after 28 days of immersion and should be classified as non-degradable. Although it has typically been reported that BCP degrades faster than HA [29], HA in previous studies have usually undergone sintering, causing it to be stable with limited degradability. This contrasts with 3DP HA, which contains numerous micropores, low crystallinity, and a nanocrystal structure resulting from the non-sintered, low-temperature process it undergoes in preparation [16]. These factors all helped 3DP HA to readily resorb, as observed. 
Wicking is the ability of a liquid to flow in narrow spaces due to capillary action and has been associated with bone regeneration of the scaffold [30]. The immediate wicking capacity of the scaffold from the host tissue can activate a natural healing cascade without additional use of exogeneous factors or progenitor cells. In this study, three types of liquid were employed to represent three contexts, namely low viscosity liquid without ions (DI water), low viscosity liquid with salt ions (PBS), and high viscosity liquid (whole blood). In the case of DI and PBS, similar wicking behaviors and rates were observed. 3DP HA showed the greatest amount of liquid absorption and fastest rate followed by Bio-Oss, RegenOss, SureOss Plus, and BoneCeramic, respectively. This observation may primarily correlate with the granule morphology of each sample, in which 3DP HA displayed a highly porous structure while RegenOss and SureOss Plus showed relatively dense structures. This is also consistent with previous reporting on the superior water absorption of 3DP HA relative to BCP [31]. The high wicking capacity has been described as a consequence of the greater porosity and lower tapped density of 3DP HA granules compared to other granules. This results in greater spaces among the granules, and water absorption of the granules is due to the capillary action of the pores in, and space among, the granules [32]. It has also been suggested that the smaller the pore size of the scaffold, the higher the fluid increase in the structure. This is consistent with our study, as the 3DP HA contained micropores with a mean pore size of $0.2 \mu \mathrm{m}$ and demonstrated a significantly higher wicking capacity than the other samples that generally contained macropores. Unlike Bio-Oss, the broken trabecular-like structure of BoneCeramic was not as effective in wicking the low-viscosity liquid. When tested with whole blood, 3DP HA also showed the greatest wicking capacity, but BoneCeramic had a more favorable wicking performance relative to RegenOss, SureOss Plus, and even Bio-Oss. This contrasted with the results from the DI and PBS tests. It is known that viscosity greatly influences capillary action and the wicking property of samples. 
Therefore, it can be inferred that the pore structure of Bio-Oss is not compatible with highviscosity liquid such as blood. It should be noted that Bio-Oss graft merely fills a bony defect and does not require wicking of blood when being implanted in an animal model [30]. In contrast, the open-channeled structure of BoneCeramic is more suitable with blood. Although previous research has reported that BCP has low hydrophilicity when tested with blood [33], the present research found that BCP could mix well with blood, and blood can be used as a vehicle for BCP to repair bone defects [27]. In summary, the high wicking ability of 3DP HA with both low- and high-viscosity liquids is a beneficial feature, as blood contains the factors and components necessary for osteogenesis [34].

Typically, agglomeration of granules when in contact with liquid is caused by a liquid film which is formed around the granules as a result of capillary action. Therefore, greater liquid absorption would normally result in a higher agglomeration volume. This was found to be generally true in terms of the granular agglomeration that occurred in this study. However, SureOss Plus showed significantly greater granular agglomeration than 3DP HA granules despite having a lower wicking capacity. This was likely due to the CMC constituent of the SureOss Plus granules, as CMC can act as adhesive to bind granules together, adding to the capillary action affecting the liquid. In contrast, 3DP HA granules formed an agglomeration with a liquid film resulting solely from capillary action. Therefore, its efficiency was lower despite its greater liquid absorption capacity. From this finding, it can be understood that a binder facilitating adhesion of granules is more capable of forming an agglomeration than binding facilitated by liquid from a capillary force alone.

A limitation of this study was that the material properties were compared by means of in vitro tests. These may not fully represent in vivo situations for which one's biological system is involved. Regardless, this study provides useful pre-clinical information comparing the properties and characteristics of bone graft granules and evaluating the possibility of 
using 3DP HA as bone graft material in alveolar cleft treatment. The material has potential for such an application due to its resemblance to allograft in terms of having high resorbability while displaying a significantly greater wicking capacity.

\section{CONCLUSION}

The results presented in this study found that 3DP HA is a favorable bone graft material candidate for treating alveolar cleft. Its resorption ability and exceptionally high wicking capacity would be beneficial to bone regeneration and tooth movement or eruption on the cleft side. In addition, its moderate granular agglomeration performance would facilitate graft handling and manipulation during placement. However, further clinical study is needed to verify the performance of 3DP HA in terms of bone regeneration performance in alveolar cleft applications.

\section{MATERIALS AND METHODS}

\subsection{Sample preparation}

3D printed hydroxyapatite was prepared as described in previous studies [13,16-17]. Briefly, a calcium sulfate-based powder mixture (Visijet PXL, 3D Systems) was loaded into a powder-based, three-dimensional printing machine (Projet 160, 3D systems) to print cylindershaped granules that were $1.0 \mathrm{~mm}$ in both diameter and thickness. These granules were transformed into hydroxyapatite by soaking them in $1 \mathrm{M}$ disodium hydrogen phosphate solution at $100{ }^{\circ} \mathrm{C}$ for $24 \mathrm{~h}$. Commercially available dental bone graft granules, including bovine xenograft (Bio-Oss, 1-2 mm, Geistlich Pharma., Wolhusen, Switzerland), freeze-dried bone allograft (FDBA) (RegenOss, 0.2-1.0 mm, Cellumed., Seoul, Korea), freeze-dried bone allograft with 5\% carboxymethyl cellulose (CMC) (SureOss Plus, 0.85-1.50 mm, Hans 
Biomed Corp., Seoul, Korea), and biphasic calcium phosphate alloplast (BCP) (BoneCeramic, 0.5-1 mm, Straumann., Basel, Switzerland), were purchased and used as control samples for comparison. Morphology was characterized by using a scanning electron microscope (JEOL JSM-7800F Prime). All the samples were gold-sputtered prior to observation.

\subsection{In Vitro Resorbability}

The experimental samples were dried in the oven at $80{ }^{\circ} \mathrm{C}$ for 1 hour before their weight was measured using a precision 4-digit balance (Mettler Toledo AB 204-S). This was recorded as the initial weight $\left(\mathrm{W}_{0}\right)$. They were then immersed in $\mathrm{pH} 7.4$ Tris(hydroxymethyl)aminomethane-buffered solution at $37^{\circ} \mathrm{C}$ for 28 days. At $1,7,14$, and 28 days, the samples were taken out, rinsed with deionized water, and dried at $80{ }^{\circ} \mathrm{C}$ for 1 hour. The dried samples were then re-weighed, and the new measurement was recorded as weight after immersion $\left(\mathrm{W}_{1}\right)$. The weight loss percentage helped to indicate the resorption of the samples, calculated using the equation

$$
\text { Weight loss }(\%)=\frac{W_{1}-W_{0}}{W_{0}} \times 100 \text {, }
$$

where:

$\mathrm{W}_{1}=$ Weight after immersion $(\mathrm{g})$

$\mathrm{W}_{0}=$ Initial weight $(\mathrm{g})$ 


\subsection{Wicking Property}

Prior to analyzing the samples' wicking property, they were dried in an oven at $80{ }^{\circ} \mathrm{C}$ for 1 hour. Wicking ability of the granules was determined by capping one end of a plastic tube ( $7 \mathrm{~mm}$ in diameter) with fine mesh before filling the tube up to a height of $70 \mathrm{~mm}$ with the granules. The capped end of the tube was then placed in contact with one of three types of liquid employed in the test: deionized water, phosphate-buffered saline, or human whole blood. The acid-citrate-dextrose (ACD) human whole blood was acquired from the blood bank at the Faculty of Medicine, Khon Kaen University, and the use of blood in this study was reviewed and approved by the Khon Kaen University Ethics Committee for Human Research (HE621104). The weight change of the liquid over time, resulting from absorption by the granules due to capillary force, was continuously recorded over 180 seconds of being in contact with each liquid. A 3-digit balance (Mettler Toledo MS 1003TS) and a video monitoring system were used to record the weight change. The liquid absorption percentage was calculated using the equation

$$
\text { Liquid absorption }(\%)=\frac{W_{A}}{W_{G}} \times 100,
$$

where:

$\mathrm{W}_{\mathrm{G}}=$ Dry weight of granules $(\mathrm{g})$

$\mathrm{W}_{\mathrm{A}}=$ Weight of absorbed liquid $(\mathrm{g})$

The liquid absorption rate of each sample was determined by the initial and late linear portions of the curve representing liquid absorption vs times. 


\subsection{Granular agglomeration}

The samples were dried in an oven at $80{ }^{\circ} \mathrm{C}$ for 1 hour to prepare them for the granular agglomeration analysis. Tapped density of the samples was firstly determined by filling a plastic tube with the weighed granules, tapping, and then measuring the height of the granules inside the tube. Tapped density was then calculated by dividing the known weight by the volume of the granules after tapping, using the formula

$$
\text { Tapped density }(\mathrm{D})=\frac{\mathrm{W}}{\mathrm{V}}
$$

where:

$\mathrm{W}=$ Weight of granules $(\mathrm{g})$

$\mathrm{V}=$ Volume after tapping $\left(\mathrm{mm}^{3}\right)$

Granular agglomeration ability of the samples was determined by forming a granular bed in the container and using a micropipette to drop on the bed a liquid droplet of $30 \mu \mathrm{L}$. As in the liquid absorption test, three types of liquid were employed, including deionized water, phosphate-buffered saline, and human whole blood. The non-agglomerated granules were recovered and their weight was compared with the initial weight of the granular bed to deduce the weight of the granular agglomeration that had formed. The volume of the agglomeration was calculated by dividing the weight of the agglomerated granules with their tapped density, expressed as

$$
\text { Agglomeration volume }=\frac{\mathrm{w}}{\mathrm{D}}
$$

where:

$\mathrm{W}=$ weight of agglomerated granule $(\mathrm{g})$

$\mathrm{D}=$ tapped density $\left(\mathrm{g} / \mathrm{mm}^{3}\right)$ 


\title{
5.5 Statistical analysis
}

All statistical analyses were performed using SPSS for windows version 22.0 (IBM Corp., Armonk, NY, USA). The Shapiro-Wilks test was used to validate the normal distribution of the data. The significance of differences in mean measurements among the sample groups was determined using an analysis of variance (ANOVA), and a pairwise multiple comparison analysis was performed by Tukey's post hoc test. The p-value was set at $\mathrm{p}<0.05$, indicating the level of significance when analyzing the differences between samples.

\author{
Abbreviations \\ HA: hydroxyapatite; 3DP HA: Three-dimensionally printed hydroxyapatite; \\ PBS: phosphate-buffered saline; $\beta$-TCP: beta-tricalcium phosphate; BCP: biphasic \\ hydroxyapatite/tricalcium phosphate ; DI: deionized water; CMC: carboxymethyl cellulose; \\ ACD: acid-citrate-dextrose
}

\section{Declarations}

\section{Ethics approval and consent to participate}

The use of blood in this study was reviewed and approved by the Khon Kaen University Ethics Committee for Human Research (HE621104).

\section{Consent for publication}

Not applicable. 


\section{Availability of data and materials}

The data used and analyzed during the current study are available from the corresponding author on reasonable request.

\section{Competing interests}

The authors declare that they have no competing interests.

\section{Funding}

This research was financially supported by research and academic services Khon Kaen University, the center of Cleft Lip-Cleft Palate and Craniofacial Deformities, Khon Kaen University under Tawanchai Royal Grant Project(Tawanchai Center) grant I62-01-08 and Mekong Health Science Research Institute, Khon Kaen University grant 4/2562.

\section{Authors' contributions}

KT collected the data, analyzed the data, summarized the data and was a major contributor in writing the manuscript.

PP collected the data, analyzed the data, summarized the data and wrote the manuscript.

JS collected the data, analyzed the data and summarized the data.

FT collected the data.

UA collected the data.

TA collected the data.

SK collected the data.

AP statistically analyzed the data. 
All authors read and approved the final manuscript.

\section{Acknowledgements}

The authors wish to thank National Metal and Materials Technology Center(MTEC, Thailand) for the use of characterization instruments. 


\section{REFERENCES}

1. Weissler EH, Paine KM, Ahmed MK, Taub PJ. Alveolar Bone Grafting and Cleft Lip and Palate: A Review. Plastic and reconstructive surgery. 2016;138(6):1287-95.

2. Browaeys H, Bouvry P, De Bruyn H. A literature review on biomaterials in sinus augmentation procedures. Clinical implant dentistry and related research. 2007;9(3):166-77.

3. Leonetti JA, Koup R. Localized maxillary ridge augmentation with a block allograft for dental implant placement: case reports. Implant Dent. 2003;12(3):217-26.

4. Lane JM, Sandhu HS. Current approaches to experimental bone grafting. Orthop Clin North Am. 1987;18(2):213-25.

5. Prolo DJ, Rodrigo JJ. Contemporary bone graft physiology and surgery. . Clin Orthop Relat Res. 1985;200:322-42.

6. Polo-Corrales L, Latorre-Esteves M, Ramirez-Vick JE. Scaffold design for bone regeneration. J Nanosci Nanotechnol. 2014;14(1):15-56.

7. Bose S, Roy M, Bandyopadhyay A. Recent advances in bone tissue engineering scaffolds. Trends in biotechnology. 2012;30(10):546-54.

8. Dorozhkin SV. Bioceramics of calcium orthophosphates. Biomaterials. 2010;31(7):1465-85.

9. Behnia H, Khojasteh A, Soleimani M, Tehranchi A, Atashi A. Repair of alveolar cleft defect with mesenchymal stem cells and platelet derived growth factors: a preliminary report. Journal of cranio-maxillo-facial surgery : official publication of the European Association for Cranio-Maxillo-Facial Surgery. 2012;40(1):2-7.

10. Hing KA, Annaz B, Saeed S, Revell PA, Buckland T. Microporosity enhances bioactivity of synthetic bone graft substitutes. J Mater Sci Mater Med. 2005;16(5):467-75. 
11. Trombetta R, Inzana JA, Schwarz EM, Kates SL, Awad HA. 3D Printing of Calcium Phosphate Ceramics for Bone Tissue Engineering and Drug Delivery. Annals of biomedical engineering. 2017;45(1):23-44.

12. Brunello G, Sivolella S, Meneghello R, Ferroni L, Gardin C, Piattelli A, et al. Powder-based 3D printing for bone tissue engineering. Biotechnology advances. 2016;34(5):740-53.

13. Suwanprateeb J, Suvannapruk W, Wasoontararat K. Low temperature preparation of calcium phosphate structure via phosphorization of 3D-printed calcium sulfate hemihydrate based material. J Mater Sci Mater Med. 2010;21(2):419-29.

14. Boskey AL. Mineralization of bones and teeth. Elements. 2007;3(387-393):387.

15. Dorozhkin SV. Nanosized and nanocrystalline calcium orthophosphates. Acta biomaterialia. 2010;6(3):715-34.

16. Thammarakcharoen F, Palanuruksa P, Suwanprateeb J. In Vitro Resorbability of Three Different Processed Hydroxyapatite. Key Engineering Materials. 2015;659:3-7.

17. Kijartorn P, Thammarakcharoen F, Suwanprateeb J, Buranawat B. The Use of Three Dimensional Printed Hydroxyapatite Granules in Alveolar Ridge Preservation. Key Engineering Materials. 2017;751:663-7.

18. LeGeros RZ. Properties of osteoconductive biomaterials: calcium phosphates. Clin Orthop Relat Res. 2002(395):81-98.

19. Sheikh Z, Abdallah MN, Hanafi AA, Misbahuddin S, Rashid H, Glogauer M. Mechanisms of in Vivo Degradation and Resorption of Calcium Phosphate Based Biomaterials. Materials (Basel). 2015;8(11):7913-25.

20. Dong Z, Li Y, Zou Q. Degradation and biocompatibility of porous nanohydroxyapatite/polyurethane composite scaffold for bone tissue engineering. Applied Surface Science. 2009;255(12):6087-91. 
21. Shiu H.T, Goss B, Lutton C, Crawford R, Xiao Y. Formation of blood clot on biomaterial implants influences bone healing. Tissue Eng Part B Rev. 2014;20:697712.

22. Parteli E.J.R, Schmidt J, Blümel C, Wirth K, Peukert W. Attractive particle interaction forces and packing density of fine glass powders. Sci Rep. 2014;4:1-7.

23. Li Q, Wang T, Zhang GF, Yu X, Zhang J, Zhou G, et al. A Comparative Evaluation of the Mechanical Properties of Two Calcium Phosphate/Collagen Composite Materials and Their Osteogenic Effects on Adipose-Derived Stem Cells. Stem cells international. 2016:1-12.

24. Benlidayi ME, Tatli U, Kurkcu M, Uzel A, Oztunc H. Comparison of bovine-derived hydroxyapatite and autogenous bone for secondary alveolar bone grafting in patients with alveolar clefts. Journal of oral and maxillofacial surgery : official journal of the American Association of Oral and Maxillofacial Surgeons. 2012;70(1):e95-e102.

25. Takemaru M, Sakamoto Y, Sakamoto T, Kishi K. Assessment of bioabsorbable hydroxyapatite for secondary bone grafting in unilateral alveolar cleft. J Plast Reconstr Aesthet Surg. 2016;69(4):493-6.

26. Rashid A, Dar S.H, Cheema J.A, Azeem M, Iftikhar M. Alveolar clefts recontruction: a comparison of autogenous bone graft versus autogenous combined with alloplastsWITH ALLOPLASTS. Pakistan Oral \& Dental Journal 2014;34(2):224-9.

27. Hing KA, Annaz B, Saeed S, Revell PA, Buckland T. Microporosity enhance bioactivity of synthetic bone graft substitute. J Mater Sci Mater Med. 2005;16(5):46775.

28. Nather AA, Yusof N, Hilmy N. Allograft Procurement, Processing And Transplantation: A Comprehensive Guide For Tissue Banks: World Scientific Publishing Company; 2010. 
29. Zhao N, Wang Y, Qin L, Guo Z, Li D. Effect of composition and macropore percentage on mechanical and in vitro cell proliferation and differentiation properties of 3D printed HA/ $\beta$-TCP scaffolds. RSC Advances. 2017;7(68):43186-96.

30. Kim S, Ahn T, Han M, Bae C, Oh D. Wicking Property of Graft Material Enhanced Bone Regeneration in the Ovariectomized Rat Model. Tissue Eng Regen Med. 2018;15(4):503-10.

31. Thammarakcharoen F, Yampakdee A, Buranawat B, Suwanprateeb J. A comparative study of granular agglomeration between 3D printed hydroxyapatite and commercial bone graft granules. Key Engineering Materials. 2019;798:83-7.

32. Oh D.S, Kim Y.J, Hong M, Han M, Kim K. Effect of capillary action on bone regeneration in micro-channeled ceramic scaffolds. Ceramics International. 2014;40(7):9583-9.

33. Trajkovski B, Jaunich M, Muller W, Beuer F. Hydrophilicity, Viscoelastic, and Physicochemical Properties Variations in Dental Bone Grafting Substitutes. Materials. 2018;11(215):1-19.

34. Shiu H.T, Goss B, Lutton C, Crawford R, Xiao Y. Formation of blood clot on biomaterial implants influences bone healing. Tissue Eng Part B Rev. 2014;20:697712. 
Figures

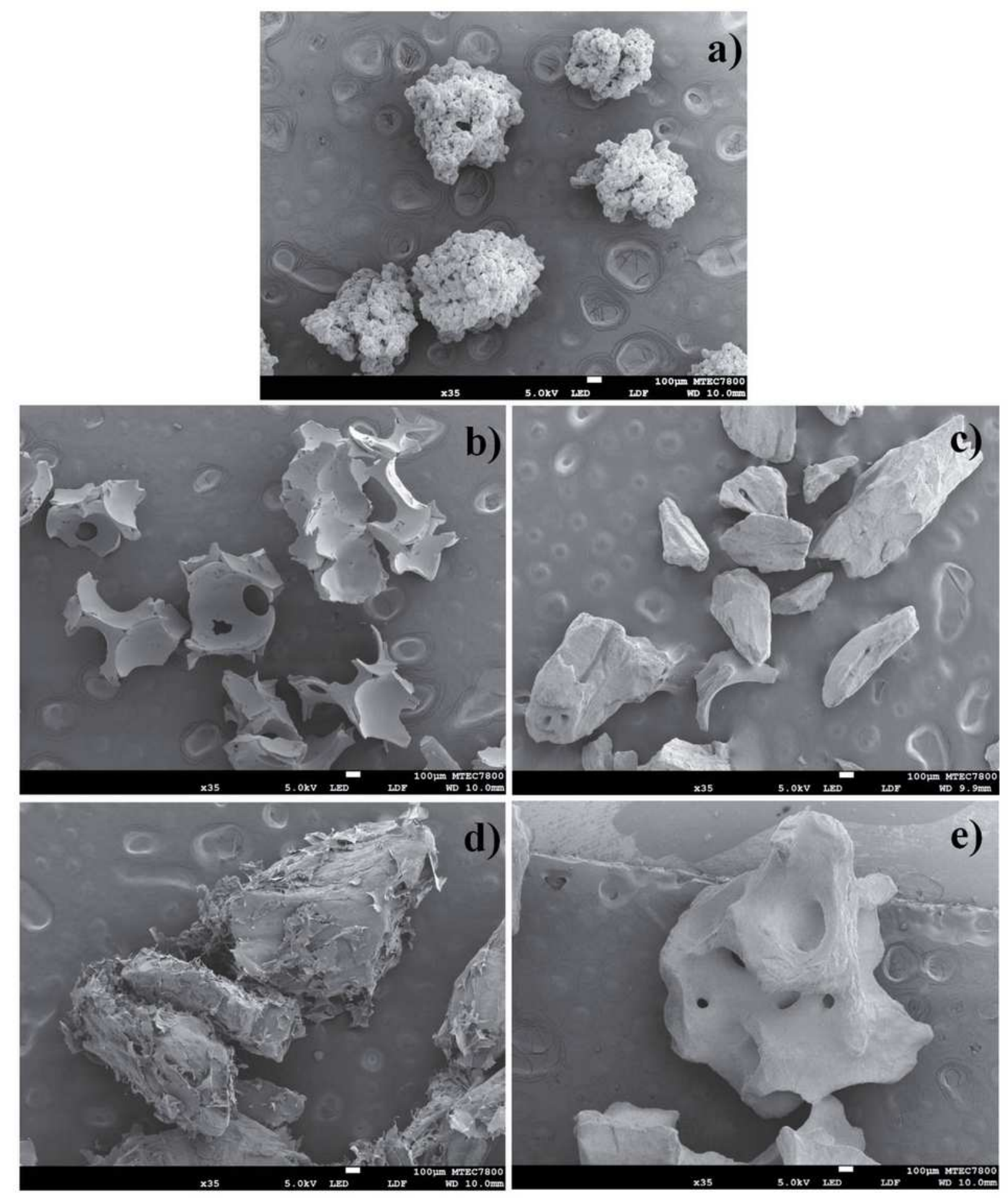

Figure 1

Morphology of the samples: a) 3DP HA; b) BoneCeramic; c) RegenOss; d) SureOss Plus; and e) Bio-Oss. 


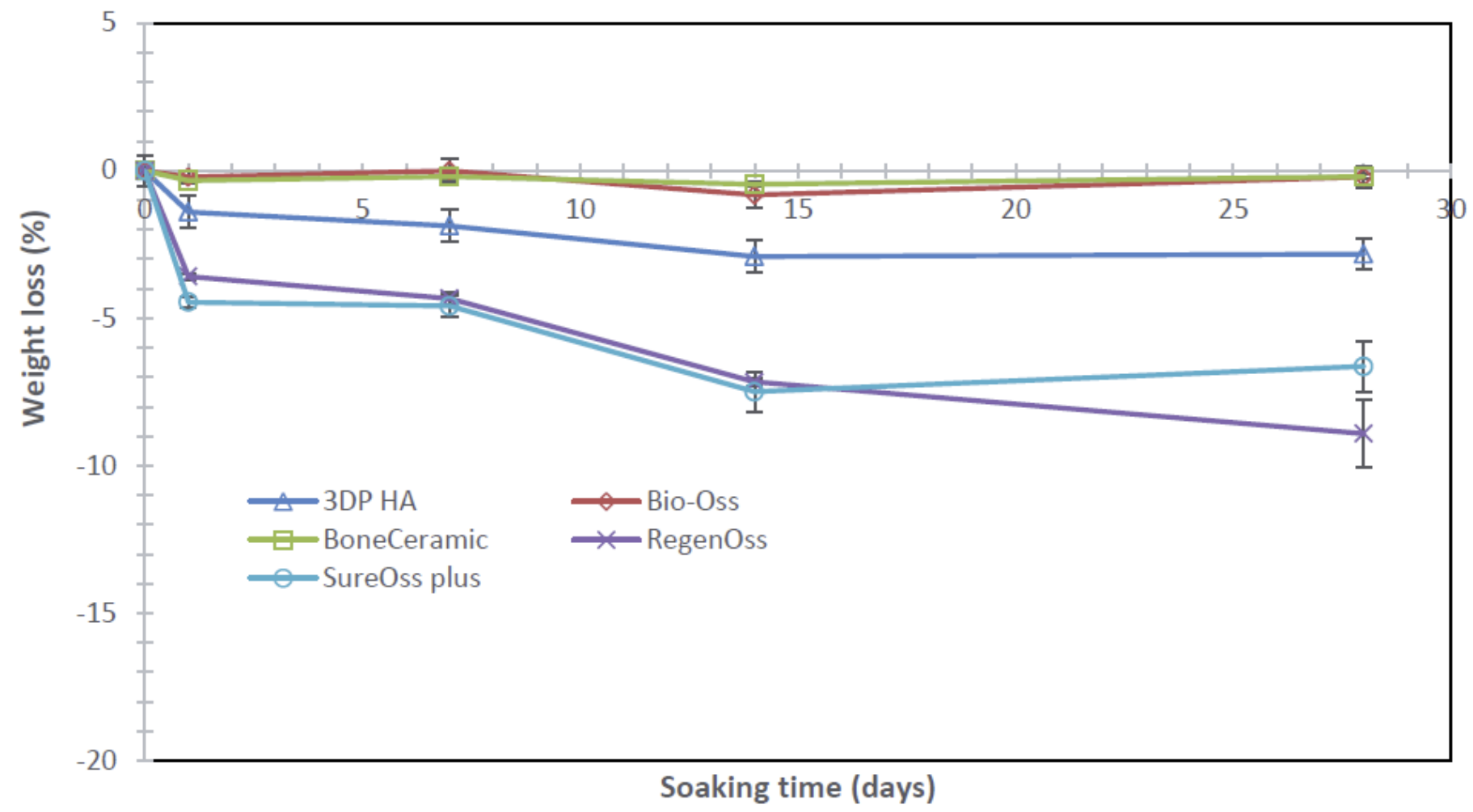

Figure 2

The change in weight loss percentage of samples at different periods after immersion in pH 7.4 TRISbuffered solution $(n=3)$. Data are presented as the mean \pm standard deviation (SD). 


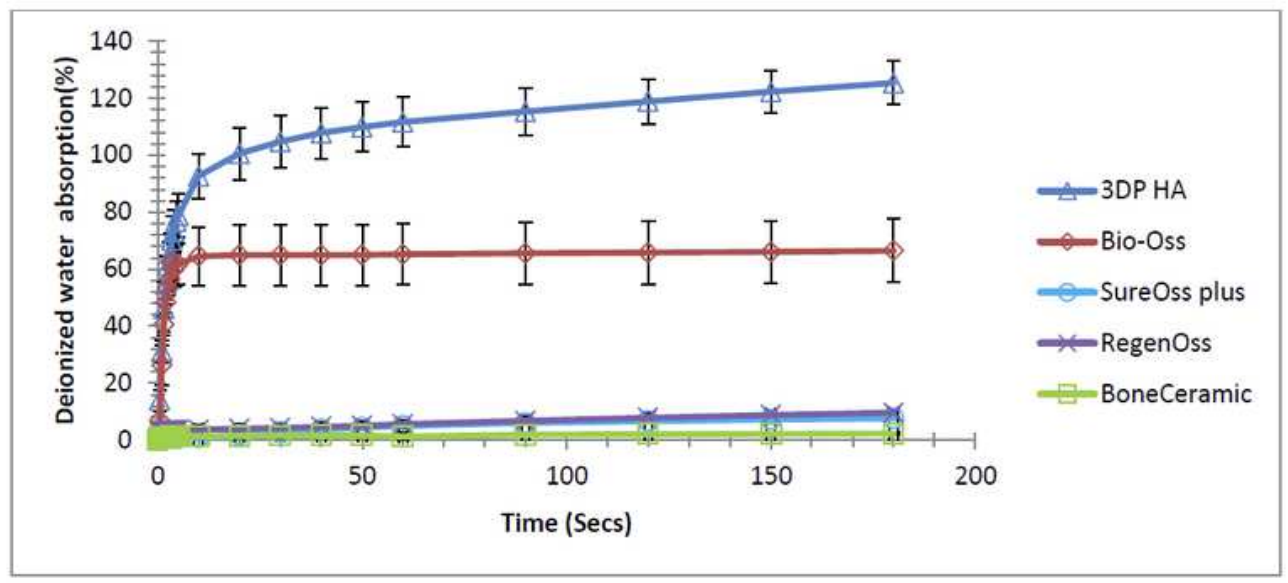

B. PBS

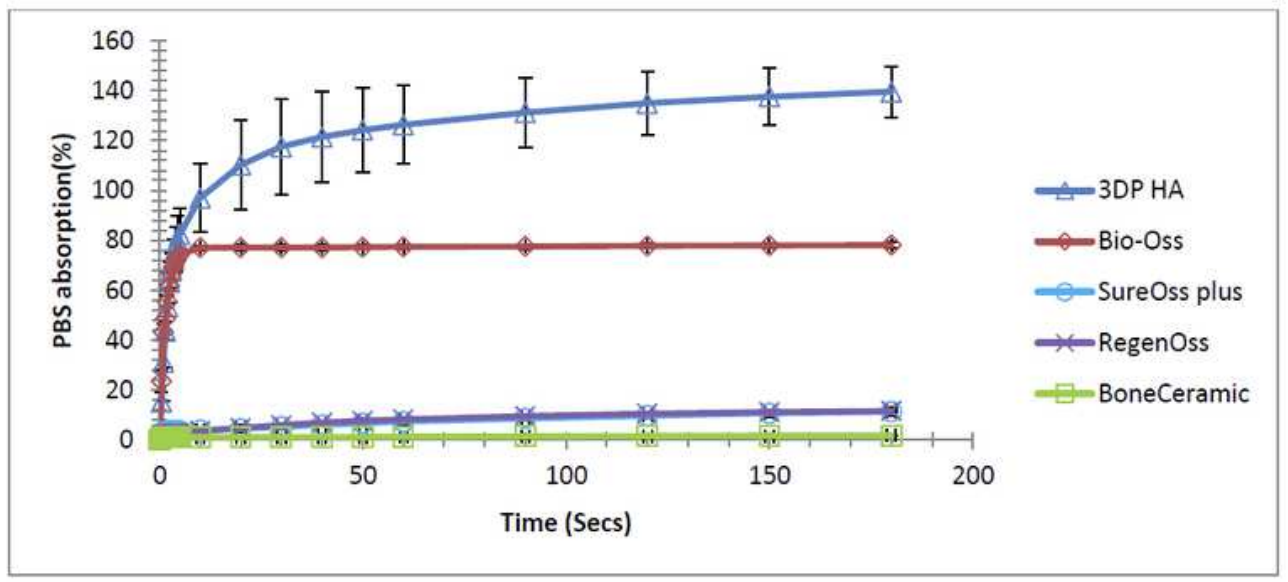

C. Whole blood

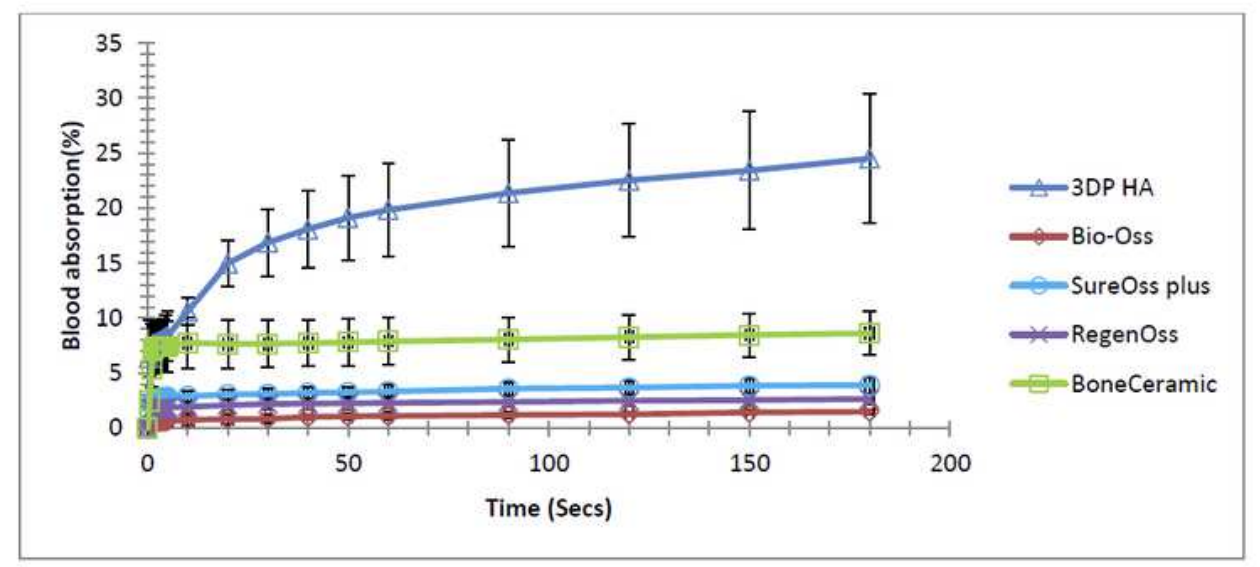

Figure 3

A, Changes in deionized water absorption percentage by samples over time $(n=3)$. Data are presented as the mean \pm standard deviation (SD). B, Changes in phosphate-buffered saline absorption percentage by samples over time $(n=3)$. Data are presented as the mean \pm standard deviation (SD). C, Changes in blood absorption percentage by samples over time $(n=3)$. Data are presented as the mean \pm standard deviation $(\mathrm{SD})$. 